\title{
Géolinguistique
}

$20 \mid 2020$

Varia

\section{Hommage à Jean Le Dû (1938-2020)}

\section{Guylaine Brun-Trigaud}

\section{(2) OpenEdition}

Journals

Édition électronique

URL : http://journals.openedition.org/geolinguistique/1916

DOI : 10.4000/geolinguistique.1916

ISSN : 2650-8176

\section{Éditeur}

UGA Éditions/Université Grenoble Alpes

\section{Édition imprimée}

ISBN : 978-2-37747-246-8

ISSN : 0761-9081

\section{Référence électronique}

Guylaine Brun-Trigaud, « Hommage à Jean Le Dû (1938-2020) », Géolinguistique [En ligne], 20 | 2020, mis en ligne le 01 décembre 2020, consulté le 24 janvier 2021. URL : http://journals.openedition.org/ geolinguistique/1916 ; DOI : https://doi.org/10.4000/geolinguistique.1916

Ce document a été généré automatiquement le 24 janvier 2021.

Géolinguistique 


\title{
Hommage à Jean Le Dû (1938-2020)
}

\author{
Guylaine Brun-Trigaud
}

1 Jean Le Dû nous a quittés le 6 mai 2020 : sa brusque disparition a laissé un grand vide dans le monde de la dialectologie, mais, bien sûr, encore plus grand pour ses proches auxquels cet hommage est également dédié.

2 Je ne retracerai pas ici le riche parcours professionnel de Jean Le Dû, d'autres l'ont fait mieux que moi (cf. André Markowicz sur Facebook ou le blog Langue bretonne.org, etc.), il s'agira juste d'évoquer quelques faits particuliers de sa carrière de géolinguiste pendant lesquels j'ai fait un peu de chemin avec lui.

\section{Le Nouvel Atlas linguistique de la Basse-Bretagne}

3 D'abord quelques mots sur l'une de ses œuvres majeures, le Nouvel Atlas linguistique de la Basse-Bretagne (NALBB). Cet atlas, conçu comme le successeur de l'Atlas de la BasseBretagne de Le Roux, a démarré dans les années 1970 sous la direction de François Falc'hun, auquel Jean Le Dû succéda lors de son départ à la retraite. D'abord hors du champ de la RCP 160 (Recherche coopérative sur programme) «Atlas linguistiques » du CNRS (1963-1977), le chantier du NALBB y fut soutenu financièrement à partir de 1973, puis inclus à part entière à partir de 1975. Les enquêtes se poursuivent jusque dans les années 1980 et dès l'origine, Jean Le Dû avait envisagé une saisie informatique des données. Cet aspect, d'abord émergeant sous le mandat du GRECO 09 «Atlas linguistiques - Parlers et cultures des régions de France » (1977-1988), deviendra crucial pour le GdR 09 (1988-1996) lorsqu'il le dirigea.

Pour ce faire, très tôt, Jean Le Dû avait fait mettre au point un outil de saisie et de cartographie automatisée avec des collègues de l'Université de Bretagne occidentale et avait commencé à effectuer de la saisie avec Bernard Tanguy. En 1987, lors de la première réunion de réflexion sur l'informatisation des atlas, il remet un rapport sur cet outil et il est même chargé d'une mission de réflexion sur cet aspect.

Ce qui aboutira au début des années 1990, à la mise en place d'un nouveau mode de saisie, l'obligeant à reprendre la totalité de son travail d'informatisation, pendant le temps de ses congés, car il était enseignant à plein temps. 
6 L'arrêt brutal du GdR 09, en 1996, le contraignit à se tourner vers d'autres financements pour assurer la publication du NALBB en 2001 (les deux volumes sont aujourd'hui épuisés).

7 Il faut ajouter que les données phonétiques du NALBB offrent la particularité d'être en API, contrairement aux autres atlas du domaine français qui ont perpétué la tradition de l'alphabet Rousselot, ce fait s'explique par l'harmonisation que Jean Le Dû souhaitait avec les autres atlas du domaine celtique.

8 Mais ces données, saisies dans un codage spécial sur 3 caractères pour chaque lettre permettant de générer la phonétique (les polices Unicode n'existaient pas encore) étaient, à leur tour, devenues inexploitables quelques années plus tard. Il fut très surpris et heureux le jour où je lui ai présenté une version de sa base convertie en Unicode: d'un seul coup, son "trésor» d'une précision extraordinaire redevenait disponible et manipulable.

\section{L'informatisation des atlas}

En 1989, à la suite de Jean Lanher, Jean Le Dû devient directeur du GdR 09, il se retrouva donc à la tête d'une entreprise qui avait reçu l'injonction de prendre le virage de l'informatisation sous peine de disparition (ce que, hélas, elle n'évita pas...), et, il faut le dire, cela s'est avéré plus difficile que prévu, car les auteurs d'atlas en cours de publication n'étaient pas tous prêts à franchir ce pas, d'autant que les moyens informatiques de l'époque n'étaient pas aussi accessibles et ergonomiques que ceux d'aujourd'hui. Deux chantiers étaient alors en cours: la saisie des données pour la poursuite de la publication des atlas et la confection d'un index regroupant la totalité de leurs données lemmatisées. En 1995, dans un courrier adressé à l'ensemble des participants du GdR et aux vacataires dont je faisais partie à l'époque, il faisait le constat du «manque de bras " pour mener à bien ces deux missions : je lui proposais alors les miens. Aussitôt, il me confia la coordination du projet des index qui hélas s'arrêta en 1999. Mais ce fut le début d'une collaboration longue et fructueuse.

\section{Lectures de l'Atlas linguistique de la France}

10 Ainsi, lors d'un colloque à Brest en 1997, Jean Le Dû me fit découvrir un trésor conservé précieusement au Centre de recherche bretonne et celtique : 2000 cartes manuscrites confectionnées par François Falc'hun et ses collaborateurs à partir de l'Atlas linguistique de la France. C'était le début de l'aventure (avec de nombreuses péripéties) qui nous mena, avec Yves Le Berre, aux Lectures de l'Atlas linguistique de la France, paru au CTHS en 2005, pour lequel j'ai réalisé plus de 800 cartes dans l'esprit initial de celles de Falc'hun, tandis que Jean Le Dû et Yves Le Berre en assuraient les commentaires.

11 C'est d'ailleurs cet ouvrage que Jean Le Dû a glissé à un certain W. Labov alors que nous participions à un colloque à Lyon en 2009, ce qui lui valut d'être invité à Philadelphie, au domicile de ce dernier. 


\section{L'Atlas linguistique des Petites Antilles}

12 À la retraite, depuis 2000, Jean Le Dû disait volontiers: «Maintenant, je peux travailler » et c'est cette énorme propension à travailler et à continuer d'œuvrer pour la dialectologie qui l'ont conduit à poursuivre ses travaux jusqu'à la fin.

13 Ainsi vers 2009, Jean Le Dû m'apprit qu'il avait saisi les données pour un atlas créole dont il avait contribué à élaborer le questionnaire et dirigé les enquêtes et pour lequel il avait besoin de quelques conseils pour faire les cartes... et évidemment, de fil en aiguille, je me suis retrouvée "embarquée " dans cette nouvelle aventure qui nous conduisit à la publication des deux volumes de l'Atlas linguistique des Petites Antilles (CTHS 2011 et 2013).

14 La cartographie de cet atlas fut assurée grâce à un logiciel que Jean Le Dû, qui tenait beaucoup à cet aspect, avait fait mettre au point en 2005 par des étudiants en informatique de l'ENST Bretagne pour l'interprétation des atlas linguistiques. Le logiciel Boumoco permettait de réaliser des cartes à symboles, mais aussi des cartes à aires. Il avait été repris et transformé pour les besoins de la publication d'Ichtyonymie bretonne (<https://ichtyo.cnrs.fr>) que Jean Le Dû avait supervisée, il nous servit pour la confection des cartes par un travail en tandem : Jean Le Dû préparait minutieusement les données et les commentaires et j'étais chargée du produit de sortie (couleurs, légendes, mise en page, etc.). Mais le plus impressionnant fut la constitution minutieuse des index qui figurent dans le second volume: près de 9000 formes lemmatisées...

15 On retrouve là son amour pour les dictionnaires et son incroyable capacité de travail, car entre-temps étaient parus Du café vous aurez? (2002 et 2011) et Le trégorrois à Plougrescant. Dictionnaire breton français (2012).

16 Sans parler de ses activités au sein de l'Atlas Linguarum Europae (ALE) et de l'Atlas linguistique roman (cf. photo) pour lequel, après m'avoir invité à participer à la synthèse sur les dénominations romanes de la pie (2018), il s'apprêtait à en recommencer une nouvelle avec Daniel Le Bris destinée au prochain volume, ni de son implication dans le renouveau des travaux de l'Atlas linguistique des côtes de l'Atlantique et de la Manche pour lequel il avait effectué les enquêtes bretonnes.

17 Parmi les derniers ouvrages parus, il faut citer Pour un néerlandais de Belgique (2020), en hommage à son ami Kas Deprez et Métamorphoses (2019) avec son complice de longue date, Yves Le Berre.

18 Enfin, ces derniers temps, il était occupé à la préparation d'un ouvrage sur le breton, fruit de ses réflexions sur un thème qui lui tenait tant à cœur et qui aurait été illustré de cartes synthétisées à partir du NALBB, mais hélas ce travail ne verra sans doute pas le jour.

19 Enfin, son aisance naturelle dans toutes sortes de langues m'a toujours impressionnée : je garde un vif souvenir d'une conversation qu'il eut avec un Gallois, ravi de rencontrer un "compatriote» en Catalogne ou de cette visite improvisée et commentée en portugais d'une église orthodoxe en compagnie d'un habitant de Lisbonne, rencontré par hasard dans la rue...

20 Plus qu'un collègue, plus qu'un modèle, je déplore la perte d'un ami très cher, toujours à l'écoute et soucieux des autres, toujours ouvert à une discussion sérieuse ou plus légère et surtout plein d'une expérience irremplaçable. 
Colloque ALiR, Ovada (Italie), 2006 : Marie-Rose Simoni-Aurembou et Jean Le Dû.

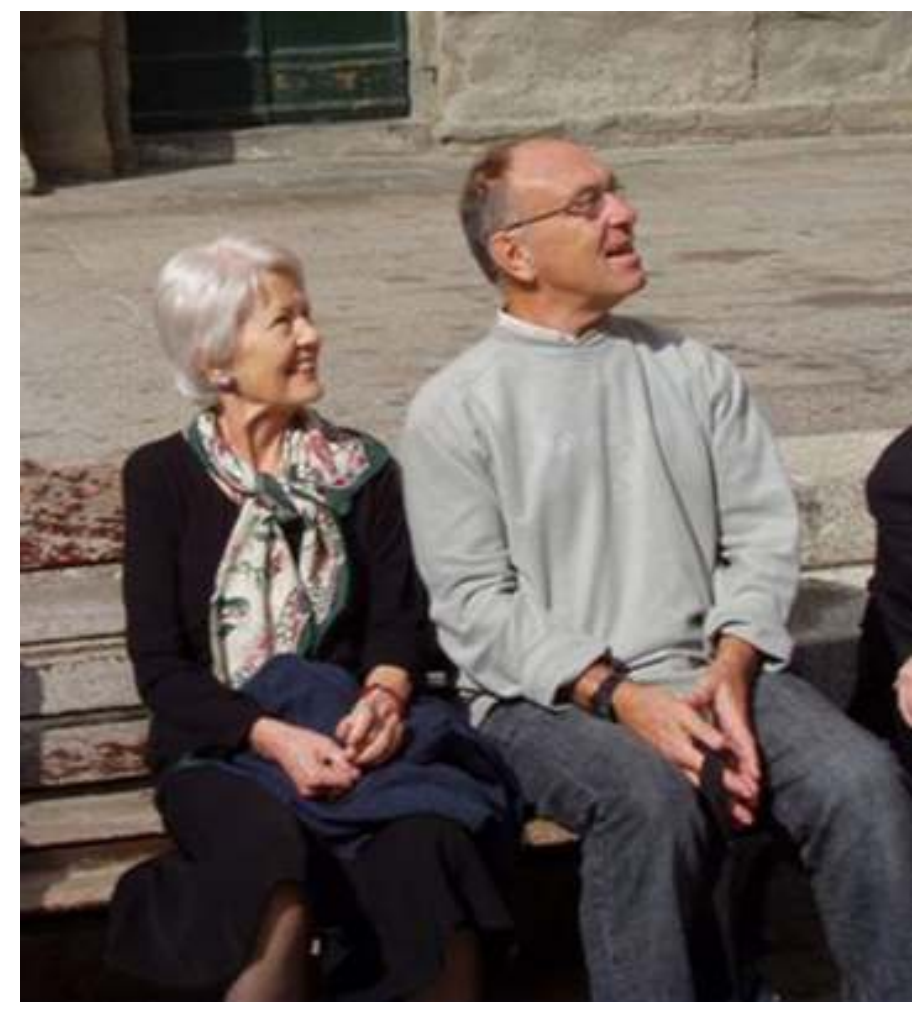

Photo de G. Brun-Trigaud.

\section{BIBLIOGRAPHIE}

BRUn-Trigaud Guylaine, LE BERRE Yves \& LE DÛ Jean, 2005, Lectures de l'Atlas linguistique de la France de Gilliéron et Edmont. Du temps dans l'espace, Paris, CTHS.

LE DÛ Jean, 2001, Nouvel Atlas linguistique de la Basse-Bretagne, Brest, CRBC, 2 vol., 600 cartes.

LE DÛ Jean, 2011, Du café vous aurez? Quand les Bretons parlent le français [2002, éd. Armeline], Brest, Emgleo Breiz.

LE Dû Jean, 2012, Le trégorrois à Plougrescant. Dictionnaire breton français, Brest, Emgleo Breiz.

LE DÛ Jean \& BRun-Trigaud Guylaine, 2011, Atlas linguistique des Petites Antilles, vol. 1, Paris, CTHS.

LE DÛ Jean \& BRun-Trigaud Guylaine, 2013, Atlas linguistique des Petites Antilles, vol. 2, Paris, CTHS.

LE DÛ Jean \& BRUN-TRIGAUD Guylaine, 2018, « Les dénominations romanes de la pie (carte et commentaires) ", dans G. Brun-Trigaud, E. Carpitelli, M. Contini et al. (éds), Atlas linguistique roman, vol. II.c., Alessandria, Edizioni dell'Orso, p. 231-252. 
LE DÛ Jean \& LE BERRE Yves, 2019, Métamorphoses. Trente ans de sociolinguistique à Brest, Brest, CRBC, coll. « Lire/Relire ».

LE Dû Jean \& LEDEGEN Gudrun, 2020, Pour un néerlandais de Belgique. Écrits d'un précurseur. Hommage à Kas Deprez, Paris, L'Harmattan.

\section{AUTEUR}

\section{GUYLAINE BRUN-TRIGAUD}

Université Côte d'Azur, CNRS, BCL, France

mgbrun@unice.fr 